\title{
Inference in Cardiovascular Modelling Subject to Medical Interventions
}

\author{
L. Mihaela Paun ${ }^{1}$, Agnieszka Borowska ${ }^{1}$, Mitchel J. Colebank ${ }^{2}$, Mette S. Olufsen ${ }^{2}$ and Dirk Husmeier ${ }^{1}$ \\ ${ }^{1}$ School of Mathematics and Statistics, University of Glasgow, Glasgow G12 8SQ, UK \\ ${ }^{2}$ Department of Mathematics, North Carolina State University, Raleigh, NC 27695, USA \\ Emails: 1.paun.1@research.gla.ac.uk; agnieszka.borowska@glasgow.ac.uk; mjcoleba@ncsu.edu; \\ msolufse@ncsu.edu; dirk.husmeier@glasgow.ac.uk;
}

\begin{abstract}
Pulmonary hypertension (PH), i.e., high blood pressure in the lungs, is a serious medical condition that can damage the right ventricle of the heart and ultimately lead to heart failure. Standard diagnostic procedures are based on right-heart catheterization, which is an invasive technique that can potentially have serious side effects. Recent methodological advancements in fluid dynamics modelling of the pulmonary blood circulation system promise to mathematically predict the blood pressure based on non-invasive measurements of the blood flow. Thus, subsequent to PH diagnostication, further investigations would no longer require catheterization. However, in order for these alternative techniques to be applicable in the clinic, accurate model calibration and parameter estimation are paramount. Medical interventions taken to combat high blood pressure (as predicted from the mathematical model) alter the underlying cardiovascular physiology, thus interfering with the parameter estimation procedure. In the present study, we have carried out a series of cardiovascular simulations to assess the reliability of cardiovascular physiological parameter estimation in the presence of medical interventions. Our principal result is that if the closed-loop effect of medical interventions is accounted for, the model calibration provides accurate parameter estimates. This finding has important implications for the applicability of cardio-physiological modelling in the clinical practice.
\end{abstract}

Keywords: Pulmonary hypertension, vasodilation, cardiovascular modelling, model calibration, statistical inference, closedloop effects, clinical decision support

\section{Introduction}

Pulmonary hypertension is defined as mean blood pressure above $20 \mathrm{mmHg}$ in the main pulmonary artery (MPA) of the lungs. It is a serious medical condition that can damage the right ventricle of the heart. The disease is characterized by a thickening and stiffening of the walls of the pulmonary arteries. This leads to increased pulmonary vascular resistance and reduced blood flow, which makes it harder for the right ventricle to pump blood through the arteries. If the right ventricle has to continually work harder, it can gradually become weaker. This can ultimately lead to heart failure.

Standard diagnostic procedures are based on right-heart catheterization. Here, a small, hollow tube called a pulmonary artery catheter is inserted into the pulmonary artery to directly measure the pressure inside the heart and pulmonary arteries [1]. This is an invasive technique, which comes with a series of potential side effects. This includes excessive bleeding due to puncture of the vein during catheter insertion, air embolism (air leaking into the heart or chest area), and cardiac tamponade (fluid buildup around the heart that affects its ability to pump blood effectively) [1]. While these complications are rare, they are serious and can result in death.

Recent methodological advancements in the fluid dynamics modelling of the pulmonary blood circulation system [3, 4, 5] have the potential to predict the blood pressure based on non-invasive measurements of the blood flow. This follows the following workflow. First, a C omputed Tomography (CT) s can of a p atient's l ung is t aken, a nd s tate of the a rt image processing techniques, as described in [6], are applied to reconstruct the patient's blood vessel network. A physical model of the blood flow in the blood vessel network is a pplied to model the vessel-specific de pendence be tween the blood flow and the blood pressure. To simplify the computational complexity of this model, the original 3-dimensional (3D) blood vessel network is mapped to a 1D graph, as shown in Figure 1. Blood flow and pressure are determined in this network by solving a 1D fluid dynamics model in e ach $\mathrm{v}$ essel. The fluid-dynamics model depends on various biophysical parameters: specifying the blood vessel network (e.g. radii), the blood characteristics (e.g. viscosity), the vessel tissue (e.g. stiffness), or the micro-vasculature dynamics (e.g. vessel network outlet). Some of these parameters, e.g. the blood's viscosity, can be directly measured, while other parameters, e.g. the blood vessel radius, can be obtained from the CT scans. However, the clinically most relevant parameters, in particular the vessel stiffness or the network outlet parameters, cannot be measured in vivo and therefore have to be inferred indirectly from the measured blood pressure and/or blood flow time series, as e.g. discussed in $[2,7,8]$. Once the parameters have been estimated, the calibrated model can be used to predict the blood pressure 


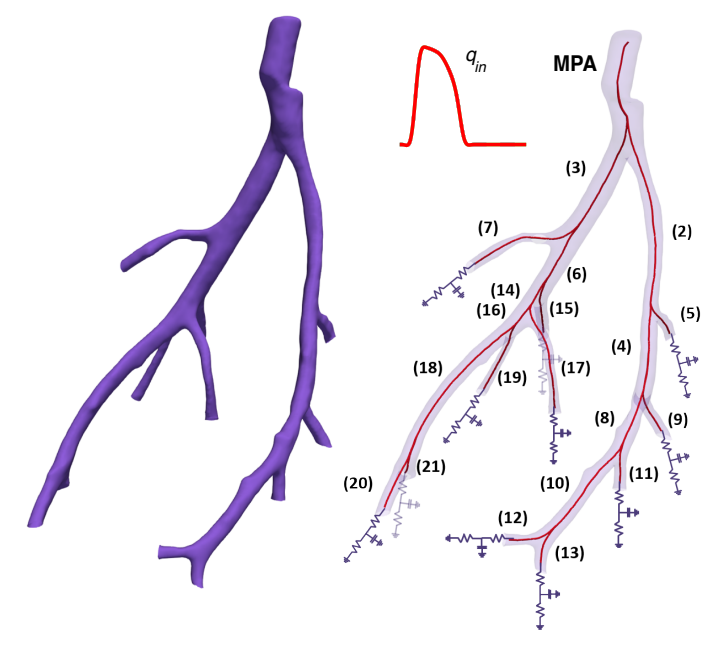

Fig. 1: Pulmonary blood vessel network. Left panel: 3D segmented blood vessel network from a micro-CT image of a healthy mouse lung. Right panel:1D directional graph of the same network with vessel numbers attached. Due to limited CT resolution, the small blood vessels at the terminal ends (micro-vasculature) are approximated by an electronic circuit (a so-called Windkessel model) consisting of two resistors and a capacitor. Inlet to main pulmonary artery (MPA): a blood flow waveform taken from ultrasound measurements.

Figure taken from our study in [2].
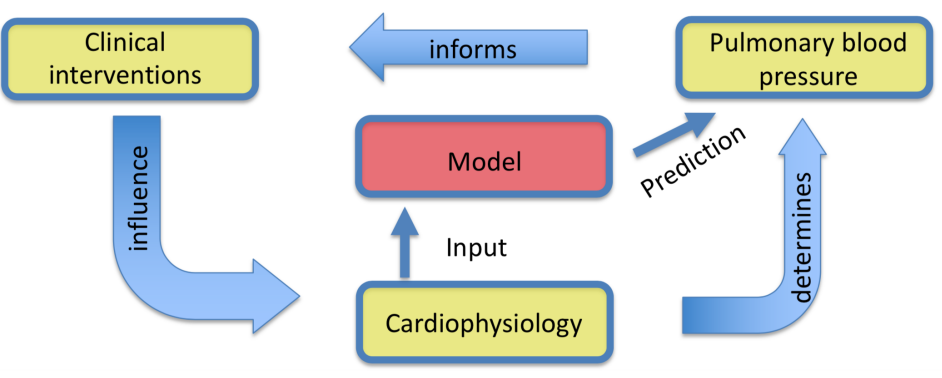

Fig. 2: Illustration of the closed-loop effect caused by clinical intervention and how it affects cardiovascular modelling. See Section 1 for a more detailed discussion.

from measurements of the blood flow. The latter can be measured non-invasively with MRI. Mathematical modelling of the fluid dynamics of the pulmonary blood circulation system thus promises to make risky invasive procedures like right-heart catherization unnecessary subsequent to $\mathrm{PH}$ diagnostication, as well as understand disease progression.

There are two methodological challenges that need to be overcome in order to make the procedure outlined above clinically viable. The first challenge is related to computational complexity. The high computational costs of numerically solving the fluid dynamics equations, which have to be carried out repeatedly during model calibration as part of an iterative parameter optimization or sampling procedure, currently render real time clinical decision making infeasible. Recent methodological advancements in statistical surrogate modelling can be used to overcome this hurdle by training a computationally cheap statistical emulator to replace the original mathematical model (the so-called simulator), as e.g. discussed in [9]. The second challenge is related to a closed-loop effect that arises when making clinical decisions and medical interventions based on predictions from the mathematical model. This is illustrated in Figure 2. The calibrated mathematical model allows a prediction of the pulmonary blood pressure based on non-invasive blood flow measurements. However, patients with a mean blood pressure in the MPA above $20 \mathrm{mmHg}$ will receive a medical intervention, most commonly in the form of vasodilator drugs. This will widen the blood vessel diameter to bring the blood pressure and vessel stiffness down. However, the consequence is a closed-loop effect that alters the physiological conditions under which the model was initially calibrated, rendering its predictions no longer valid [10]. In principle, a new CT scan could be taken, and the model could be recalibrated based on new vessel network parameters. However, this is not feasible in the clinical practice, as taking a follow-up CT scan 
with a subsequent reconstruction of the blood vessel network is not only a time-consuming complex procedure, but would also expose the patient to a new dose of radiation. Following up on the paper we presented at last year's ICSTA conference [11], where we demonstrated that ignoring the closed-loop effect can lead to a systematic estimation bias, in the current study we investigate if a recalibration of the mathematical model is feasible based on blood flow measurements alone, without the need for another CT scan.

\section{Mathematical model}

This study uses our previously developed 1D fluid-dynamics model [5], predicting blood pressure, blood flow, and crosssectional area in the large pulmonary arteries (shown in Figure 1). For each vessel, the fluid dynamics can be described by the following partial differential equations, which have been derived under various physical assumptions discussed in [5] (e.g. blood is incompressible and flow is Newtonian, laminar and axisymmetric, and has no swirl):

$$
\frac{\partial A}{\partial t}+\frac{\partial q}{\partial x}=0, \quad \frac{\partial q}{\partial t}+\frac{\partial}{\partial x}\left(\frac{q^{2}}{A}\right)+\frac{A}{\rho} \frac{\partial p}{\partial x}=-\frac{2 \pi \mu r}{\rho \delta} \frac{q}{A},
$$

Here $x(\mathrm{~cm})$ and $t(\mathrm{~s})$ denote the spatial and temporal coordinates, and $p=\tilde{p} / \mathrm{conv}(\mathrm{mmHg})$ denotes pressure $\left(\tilde{p}\left(\mathrm{~g} / \mathrm{cm} / \mathrm{s}^{2}\right)\right.$ is in cgs units and conv $=1333.22\left(\mathrm{mmHg} /\left(\mathrm{g} / \mathrm{cm} / \mathrm{s}^{2}\right)\right)$ is a conversion factor). The blood density $\rho=1.055 \mathrm{~g} / \mathrm{ml}$ and viscosity $\mu=0.049 \mathrm{~g} /(\mathrm{cm} \mathrm{s})$ are set to fixed values from the physiological literature [12]. $\delta=\sqrt{\mu T / 2 \pi \rho}(\mathrm{cm})$ is the boundary-layer thickness of the velocity profile. Vessel radius and cross-sectional area are $r(x, t)(\mathrm{cm})$ and $A(x, t)=\pi r(x, t)^{2}\left(\mathrm{~cm}^{2}\right)$, respectively. The volumetric blood flow rate $q(\mathrm{ml} / \mathrm{s})$ is modelled as a Stokes boundary layer velocity profile, as discussed in [5]. To close the system of equations, we add a constitutive pressure-area relation:

$$
p=\frac{4}{3} \chi\left(\sqrt{\frac{A}{A_{0}}}-1\right)
$$

where $\chi$ is the blood vessel wall stiffness, and $A_{0}=\pi r_{0}^{2}\left(\mathrm{~cm}^{2}\right)$ is the unstressed vessel area. At the inlet to the network (shown in Figure 1], we specify the blood flow (taken from measurements). Similar to previous studies [3, 6, 13] we assume flow conservation and pressure continuity

$$
p_{p}(L, t)=p_{d_{1}}(0, t)=p_{d_{2}}(0, t), \quad q_{p}(L, t)=\sum_{i=1}^{2} q_{d_{i}}(0, t),
$$

where $p$ denotes the parent vessel, $d_{1}$ and $d_{2}$ are the daughter vessels, and $L(\mathrm{~cm})$ is the vessel length. The micro-circulation is represented by a three-element Windkessel model (an RCR circuit) relating pressure and flow as

$$
\frac{d p(L, t)}{d t}-R_{1} \frac{d q(L, t)}{d t}=q(L, t)\left(\frac{R_{1}+R_{2}}{R_{2} C}\right)-\frac{p(L, t)}{R_{2} C}
$$

where $R_{1}, R_{2}(\mathrm{~g} / \mathrm{cm} / \mathrm{ml})$ are resistances, and $C(\mathrm{ml} \mathrm{cm} \mathrm{s} / \mathrm{g})$ is the capacitance.

The haemodynamic model has three types of parameters specifying blood characteristics, vessel tissue properties (stiffness), and micro-vasculature fluid dynamics. As previously mentioned, blood viscosity and density values are taken from the literature [12] and assumed constant. The blood vessel stiffness has to be inferred statistically, as described Section 3 . Finally, micro-vasculature parameters are prescribed in each terminal vessel's boundary condition. To model the micro-vasculature fluid dynamics, three boundary condition parameters $\left(R_{1}, R_{2}, C\right)$ are specified for each terminal vessel. The network in Figure 1 has 11 terminal vessels giving a total of 33 parameters. These parameters are not practically identifiable. To reduce parameter dimensionality, the standard procedure, discussed e.g. in [5, 6], is to introduce factors $\left(r_{1}, r_{2}, c\right)$ scaling the nominal (initial) Windkessel parameters by

$$
R_{1}^{j}=r_{1} R_{01}^{j}, \quad R_{2}^{j}=r_{2} R_{02}^{j}, \quad C^{j}=c C_{0}^{j},
$$


where $R_{01}^{j}, R_{02}^{j}, C_{0}^{j}$ are the nominal values for the $j^{\text {th }}$ terminal vessel, $R_{1}^{j}, R_{2}^{j}, C^{j}$ are the adjusted (estimated) values for the $j^{\text {th }}$ terminal vessel. $r_{1}, r_{2}, c$ are the scaling factors, common to all terminal vessels, and estimated from the available data, as discussed in Section 3. Here, $R_{01}^{j}, R_{02}^{j}, C_{0}^{j}$ are computed using the junction conditions and Poiseuille's flow, as described in detail in our previous studies [5, 6],

$$
R_{\mathrm{T}}^{j}=\frac{\bar{p}}{\bar{q}^{j}}, \quad R_{01}^{j}=0.2 R_{\mathrm{T}}^{j}, \quad R_{02}^{j}=0.8 R_{\mathrm{T}}^{j}, \quad C_{0}^{j}=\frac{\tau}{R_{\mathrm{T}}^{j}},
$$

where $R_{\mathrm{T}}^{j}$ is the total resistance for the $j^{\text {th }}$ terminal vessel, $\bar{p}$ is the mean MPA pressure (obtained with right-heart catheterization during the PH diagnostication process), $\bar{q}^{j}$ is the mean flow in the $j^{\text {th }}$ terminal vessel, and $\tau$ is a constant pressure diastolic decay time.

Since the PDEs in equations (1) and (2) subject to the boundary conditions (3)-(6) have no closed-form solution, they have to be integrated numerically, for which a Lax-Wendroff scheme [14] was used in the present work.

\section{Statistical inference}

Under the assumption of independent and identically normally distributed errors with noise variance $\sigma^{2}$, the likelihood function is given by

$$
p\left(\mathbf{y} \mid \boldsymbol{\theta}, \sigma^{2}\right)=\left(\frac{1}{\sqrt{2 \pi \sigma^{2}}}\right)^{K n} \exp \left(-\frac{E(\boldsymbol{\theta})}{2 \sigma^{2}}\right),
$$

where

$$
E(\boldsymbol{\theta})=(\mathbf{y}(\mathbf{t})-\mathbf{m}(\boldsymbol{\theta}, \mathbf{t}))^{\mathrm{T}}(\mathbf{y}(\mathbf{t})-\mathbf{m}(\boldsymbol{\theta}, \mathbf{t}))=\sum_{k=1}^{K} \sum_{i=1}^{n}\left(y_{k}\left(t_{i}\right)-m_{k}\left(\boldsymbol{\theta}, t_{i}\right)\right)^{2}
$$

is the standard sum-of-squares distance between the clinical data $\mathbf{y}$ and the model predictions $\mathbf{m}(\boldsymbol{\theta}, \mathbf{t})$. In the present study, the clinical data $y_{k}\left(t_{i}\right)$ are the blood flow rates measured in $K=3$ blood vessels (MPA and its two daughter vessels) over a time course $\mathbf{t}=\left(t_{1}, \ldots, t_{n}\right)$, and $m_{k}\left(\boldsymbol{\theta}, t_{i}\right)$ are the corresponding predictions from the mathematical model described in Section 2 This model depends on the parameter vector $\boldsymbol{\theta}=\left(\chi, r_{1}, r_{2}, c\right)$, as discussed in Section 2 We follow a Bayesian inference procedure and sample $\boldsymbol{\theta}$ from the posterior distribution with Markov chain Monte Carlo (MCMC), using the Adaptive Metropolis (AM) algorithm [15]. The prior distributions of the parameters are chosen to ensure that the blood pressure profiles stay in physiologically realistic regimes; details can be found in Table 2 of [2].

\section{Simulation study}

Mathematical cardiovascular modelling is not yet commonly used in the clinic; for that reason we resort to a simulation study to assess its clinical viability. We start with the blood vessel geometry obtained from a CT scan in a healthy mouse, taken from our previous studies [3, 4, 5, 6]. Next, we simulate blood flow and pressure using the haemodynamic model of Section 2 with physiologically realistic parameters following the study of [4]. The parameter values are reported in Figure 3 and were selected based on the hypertensive pressure, which influences the initial nominal Windkessel parameter values (eqns (5) and (6)) and the vessel stiffness value. To replicate the clinical data acquisition process, we obtain blood flow rates in the MPA and its two daughter vessels from the middle location point at $n$ time points during the cardiac pump cycle, choosing $n$ such that it

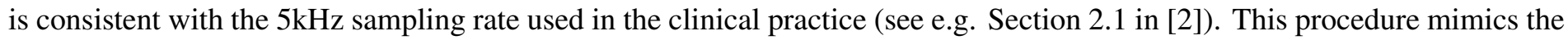
clinical non-invasive measurement with MRI. Now, to imitate the effect of a clinical intervention, we upscale the blood vessel radii by a scaling factor $\lambda>1$ :

$$
r \rightarrow \lambda r
$$

This resembles the effect of a vasodilator, which would be administered in response to a critically high mean pressure in the MPA. We note that the upscale of the blood vessel radii determines a change in the nominal Windkessel parameters $R_{01}^{j}, R_{02}^{j}, C_{0}^{j}$ 


\begin{tabular}{|c|c|c|}
\hline Pulmonary hypertension & All vessels vasodilation & Terminal vessels vasodilation \\
\hline 25.5 & 12.73 & 13.66 \\
\hline
\end{tabular}

Table 1: Mean pressure in the main pulmonary artery under hypertensive conditions, and post dilation of (1) all blood vessels, and (2) terminal vessels. The pressure prediction was obtained by numerically solving equations (1, 6), as described in Section 2 with the biophysical parameter values reported in Figure 3 .

in equation (5) and (6) (see [5] for details on how the nominal Windkessel parameters depend on the vessel radii), as follows:

$$
R_{01}^{j, \mathrm{NEW}}=\frac{R_{01}^{j, \mathrm{OLD}}}{\lambda^{4}}, \quad R_{02}^{j, \mathrm{NEW}}=\frac{R_{02}^{j, \mathrm{OLD}}}{\lambda^{4}}, \quad C_{0}^{j, \mathrm{NEW}}=\lambda^{4} C_{0}^{j, \mathrm{OLD}},
$$

where $R_{01}^{j, \mathrm{OLD}}, R_{02}^{j, \mathrm{OLD}}$ and $C_{0}^{j, \mathrm{OLD}}$ are the nominal resistances and capacitance parameters prior to vasodilation and $R_{01}^{j, \mathrm{NEW}}$, $R_{02}^{j, \mathrm{NEW}}$ and $C_{0}^{j, \mathrm{NEW}}$ are the nominal Windkessel parameters post vasodilation.

We carry out two different vasodilation simulations. In the first simulation, we assume the vasodilator to affect all blood vessels equally, and upscale all vessel radii by the same factor $\lambda$. However, there is increasing evidence that vasodilators mainly dilate small downstream blood vessels [16]. For that reason we carry out a second vasodilation simulation, where the effect of the vasodilator is restricted to the terminal vessels. For the chosen biophysical parameter values (reported in Figure 3), in Table 1 we illustrate the mean MPA pressure prior to vasodilation $(\lambda=1)$, as well as post vasodilation with both vasodilation methods. If another CT scan could be carried out, the new blood vessel radii after the medical intervention would be known, and parameter estimation would follow the procedure presented in our previous work [2, 7, 8]. However, as explained in Section 1, this is not a procedure embraced in the clinical practice. Ignoring the effect of the vasodilator and using the same blood vessel geometry as obtained from the original CT scan leads to a systematic estimation bias, as we demonstrated at last year's ICSTA conference [11]. The questions to be addressed in the present study are as follows: Can the estimation bias be avoided when explicitly including the vasodilation effect $\lambda$ in the model, i.e. when including $\lambda$ in an augmented parameter vector

$$
\tilde{\boldsymbol{\theta}}=(\boldsymbol{\theta}, \lambda)=\left(\chi, r_{1}, r_{2}, c, \lambda\right) ?
$$

Can the parameter vector $\tilde{\boldsymbol{\theta}}$ be reliably inferred based on blood flow measurements alone, without needing another CT scan?

\section{Results}

The results of our study are shown in Figures 3 and 4 . Figure 3 shows the posterior probability distribution of the five parameters inferred: the blood vessel stiffness parameter $\chi$, the micro-vasculature fluid dynamics parameters, i.e. Windkessel element parameters $r_{1}, r_{2}$, and $c$, and the vasodilation effect $\lambda$. We obtained these plots by running MCMC simulations with AM (100,000 samples were collected), applying standard convergence diagnostics based on the Rubin-Gelman potential scale reduction factor, and discarding a burn-in phase which failed the convergence test (first 10,000 s amples). We then applied a kernel density estimator to the parameters sampled during the equilibrium phase of the MCMC simulation, where the bandwidth was optimized with the method described in [17]. The plots show that the true parameters, indicated by solid vertical lines, are always well recovered, being close to the centre of the posterior distributions. Next, we inserted the sampled parameters back into the mathematical model described in Section 2, equations (1-6), to predict the temporal blood pressure profile during the cardiac pump c ycle. Recall that this quantity cannot be measured non-invasively, and that it is the ultimate objective of the cardiovascular modelling exercise to predict the blood pressure profile based on non-invasive measurements of the blood flow. For each sampled parameter vector $\tilde{\boldsymbol{\theta}}$, we numerically solve equations (1-6), as described in Section 2; hence the posterior sample of the parameter vectors $\tilde{\boldsymbol{\theta}}$ induces a posterior sample of blood pressure profiles. This distribution can be summarized by the posterior median, shown by the red dashed line in Figure 4, and the 95\% posterior credible intervals, shown by the green lines. Since this is a simulation study, the true pressure profile is known, as shown by the black solid lines. Figure 4 compares the true pressure profile with the posterior median and the $95 \%$ posterior credible intervals for the MPA (top row) and its two daughter vessels (middle and bottom rows). This comparison is repeated for both simulations of the vasodilation effect: the assumption of the vasodilator equally affecting all blood vessels (left column), and the assumption 

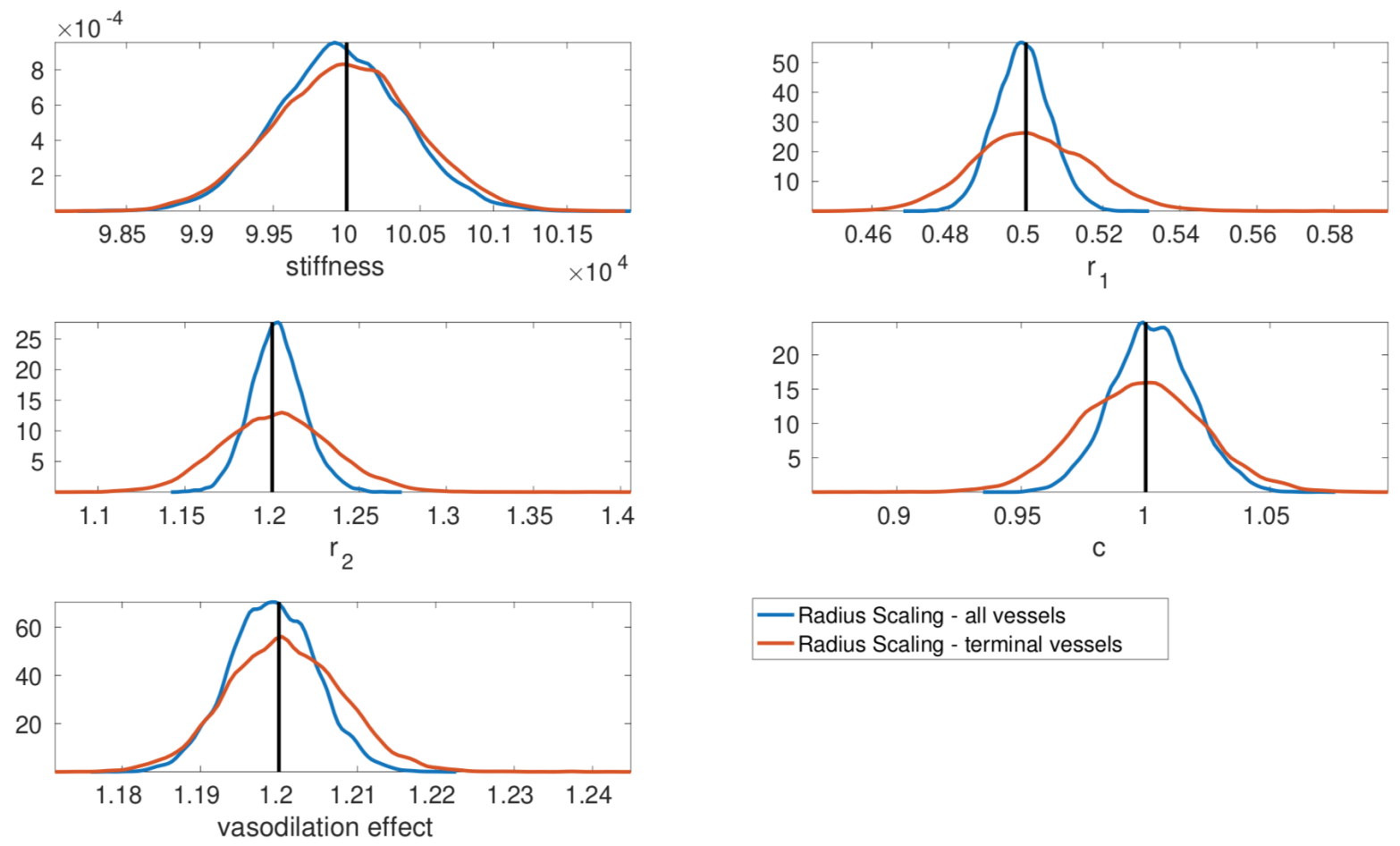

- Radius Scaling - all vessels
- Radius Scaling - terminal vessels

Fig. 3: Posterior probability distributions of the parameters. The figure shows the estimated marginal posterior probability densities of the blood vessel stiffness parameter $\chi$, the micro-vasculature fluid dynamics parameters, i.e. Windkessel element parameters $r_{1}, r_{2}$, and $c$, and the vasodilation effect $\lambda$. The plots were obtained by applying a kernel density estimator to the samples obtained from the MCMC simulations after burn-in. Vertical line: True parameter values. Blue lines: Vasodilation affecting all blood vessels. Red lines: Vasodilation affecting only the terminal blood vessels.

of the vasodilator only affecting the terminal blood vessels (right column).

We find that the predicted posterior median pressure profile is always very close to the true pressure profile, and that the true pressure profile is consistently included in the $95 \%$ posterior credible intervals. This suggests that the cardiovascular and treatment effect parameters, as well as the blood pressure profile can be accurately estimated from the blood flow profiles alone, without the need for another CT scan. We note that the posterior credible intervals are slightly broader in the first vasodilation simulation (left column) than in the second (right column). The reason for that is that the vasodilation effect $\lambda$ is itself an unknown parameter that has to be inferred. This inference comes with its own intrinsic posterior estimation uncertainty. This uncertainty has a larger leverage on the blood pressure profile when the vasodilator affects all blood vessels than when it only affects the terminal vessels.

\section{Conclusions}

Cardiovascular modelling has great promise for predicting pulmonary hypertension from non-invasive blood flow measurements, dispensing with the need for invasive right-heart catheterization. Accurate model calibration is paramount for this approach to be viable in the clinic, though. In clinical applications, the prediction of high pulmonary blood pressure above a critical threshold will trigger the administration of vasodilators, whose effect is the increase of the vessel diameter. As illustrated in Figure 2, this causes a closed-loop effect, whereby the prediction from the model causes an action that alters the conditions under which the original prediction was obtained [10]. At last year's ICSTA conference, we demonstrated that ignoring this closed-loop effect leads to a systematic parameter estimation and blood pressure prediction bias [11]. This bias could in principle be avoided if the cardiovascular model could be recalibrated based on another CT scan, but this is not an acceptable clinical procedure (due to a patient's excessive exposure to radiation). Our present study has demonstrated that the estimation and prediction bias can be avoided by explicitly modelling the vasodilation effect, via transformation (9) 

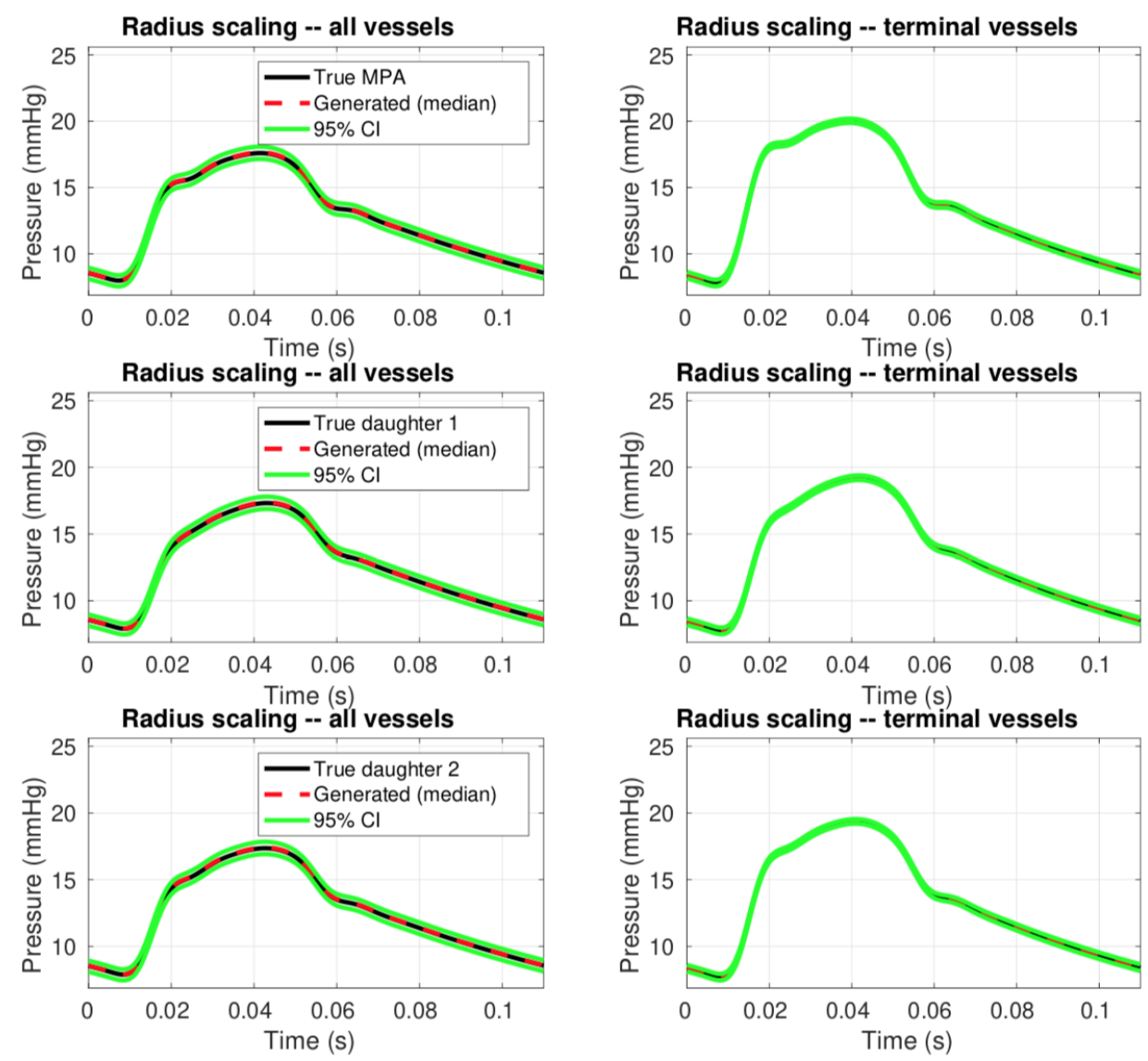

Fig. 4: Blood pressure prediction subject to vasodilation. Left column: Simulation of vasodilation where the vasodilator is assumed to affect all blood vessels equally. Right column: Alternative simulation of vasodilation where the vasodilator is assumed to affect only the terminal blood vessels. Top row: Main pulmonary artery. Middle row: Daughter 1 vessel. Bottom row: Daughter 2 vessel. Black line: True blood pressure profile during one period of the cardiac pump c ycle. Dashed red line: Posterior median prediction. Green lines: $95 \%$ posterior credible interval.

and equation (11), and that model recalibration at a high degree of accuracy is feasible based on blood flow measurements alone, without the need for another CT scan. This is an important finding, as blood flow me asurements can be obtained non-invasively via MRI, and our study thus demonstrates the huge potential that cardiovascular modelling has for the clinical practice.

Our study has a few limitations. First, the PDEs used the hypertensive flow (i.e. prior to vasodilation) as inlet boundary conditions; future studies will use the inflow in the healthy range (i.e. post-vasodilation). Second, we have only accommodated for the acute (short-term) vasodilation effect, future work will include mimicking long-term effect by a decrease of the stiffness value used to simulate the data. Third, we have not allowed for model mismatch. By directly simulating blood pressure and flow profiles from the mathematical model of Section 2, our approach does not allow for cardiovascular processes that deviate from the model of equations (1-6), e.g. due to additional physiological complexity not captured by the mathematical model. In a recent study [2], we have investigated how such unknown additional physiological complexity can be systematically integrated into the statistical inference procedure, and allowing for model discrepancy in the context of investigating the closed-loop effect of medical interventions is an important and highly topical future research direction.

\section{Acknowledgments}

This work has been funded by EPSRC, grant reference numbers EP/R018634/1 (research centre for closed-loop data science), EP/S030875/1 (research centre for multiscale soft-tissue mechanics), and EP/T017899/1 (research hub for statistical inference 
in complex cardiovascular and cardiomechanic systems). Olufsen is supported by the National Science Foundation (NSF), grant reference numbers NSF-DMS 1615820 and NSF-DMS 1246991. Colebank is supported by the American Heart Association (AHA), grant reference number 19PRE34380459.

\section{References}

[1] S. Rosenkranz and I. R. Preston, "Right heart catheterisation: best practice and pitfalls in pulmonary hypertension," European Respiratory Review, vol. 24, no. 138, pp. 642-652, 2015.

[2] L. M. Paun, M. Colebank, M. S. Olufsen, N. A. Hill, and D. Husmeier, "Assessing model mismatch and model selection in a Bayesian uncertainty quantification analysis of a fluid-dynamics model of pulmonary blood circulation," Journal of the Royal Society Interface, vol. 17, no. 173, p. 20200886, 2020.

[3] M. Umar Qureshi, G. Vaughan, C. Sainsbury, M. Johnson, C. Peskin, M. Olufsen, and N. Hill, "Numerical simulation of blood flow and pressure drop in the pulmonary arterial and venous circulation," Biomechanics and modeling in mechanobiology, vol. 13, no. 5, pp. 1137-1154, 2014.

[4] M. Qureshi, M. Haider, N. Chesler, and M. Olufsen, "Simulating effects of hypoxia on pulmonary haemodynamics in mice.," in Proc. 5th International Conference on Computational \& Mathematical Biomedical Engineering (CMBE 2017), vol. 1, pp. 271-274, Zeta Computational Resources Ltd., 2017.

[5] M. U. Qureshi, M. J. Colebank, L. M. Paun, L. E. Fix, N. Chesler, M. A. Haider, N. A. Hill, D. Husmeier, and M. S. Olufsen, "Hemodynamic assessment of pulmonary hypertension in mice: a model-based analysis of the disease mechanism," Biomechanics and Modeling in Mechanobiology, vol. 18, pp. 219-243, 2018.

[6] M. Colebank, L. Paun, M. Qureshi, N. Chesler, D. Husmeier, M. Olufsen, and L. Ellwein, "Influence of image segmentation on one-dimensional fluid dynamics predictions in the mouse pulmonary arteries," Journal of the Royal Society Interface, vol. 16, no. 159, p. 20190284, 2019.

[7] L. M. Paun, M. U. Qureshi, M. Colebank, N. A. Hill, M. S. Olufsen, M. A. Haider, and D. Husmeier, "MCMC methods for inference in a mathematical model of pulmonary circulation," Statistica Neerlandica, vol. 72, pp. 306-338, 2018.

[8] L. M. Paun, M. Colebank, M. U. Qureshi, M. Olufsen, N. Hill, and D. Husmeier, "MCMC with delayed acceptance using a surrogate model with an application to cardiovascular fluid dynamics," Proceedings of the International Conference on Statistics: Theory and Applications (ICSTA 2019), no. 28, 2019.

[9] L. M. Paun and D. Husmeier, "Markov chain Monte Carlo with Gaussian processes for fast parameter estimation and uncertainty quantification in a 1D fluid-dynamics model of the pulmonary circulation," International Journal for Numerical Methods in Biomedical Engineering, p. e3421, 2020.

[10] M. J. Colebank, A. L. Colunga, M. King, C. Schell, M. Sheldon, M. Kharbat, R. Sternquist, and M. S. Olufsen, "Parameter inference in a computational model of hemodynamics in pulmonary hypertension," 2021.

[11] D. Husmeier and L. M. Paun, "Closed-loop effects in cardiovascular clinical decision support," Proceedings of the International Conference on Statistics: Theory and Applications (ICSTA 2020), no. 128, 2020.

[12] U. Windberger, A. Bartholovitsch, R. Plasenzetti, K. J. Korak, and G. Heinze, "Whole blood viscosity, plasma viscosity and erythrocyte aggregation in nine mammalian species: Reference values and comparison of data," Experimental Physiology, vol. 88, no. 3, pp. 431-440, 2003.

[13] M. Olufsen, C. S. Peskin, W. Kim, E. M. Pedersen, A. Nadim, and J. Larsen, "Numerical simulation and experimental validation of blood flow in arteries with structured-tree outflow conditions," Annals of Biomedical Engineering, vol. 28, pp. 1281-1299, 2000.

[14] P. Lax and B. Wendroff, "Systems of conservation laws," Communications on Pure and Applied Mathematics, vol. 13, no. 2, pp. 217-237, 1960.

[15] H. Haario, E. Saksman, and J. Tamminen, "An adaptive Metropolis algorithm," Bernoulli, vol. 7, no. 2, pp. 223-242, 2001.

[16] "Effect of acute arteriolar vasodilation on capacitance and resistance in pulmonary arterial hypertension," Chest, vol. 147, no. 4, pp. 1080 - 1085, 2015.

[17] A. W. Bowman and A. Azzalini, Applied Smoothing Techniques for Data Analysis. New York: Oxford University Press Inc., 1997. 